\title{
Telecentros en España: redes y contenidos
}

\author{
Por Ana-María Morales-García, Mercedes Caridad-Sebastián y Fátima García-López
}

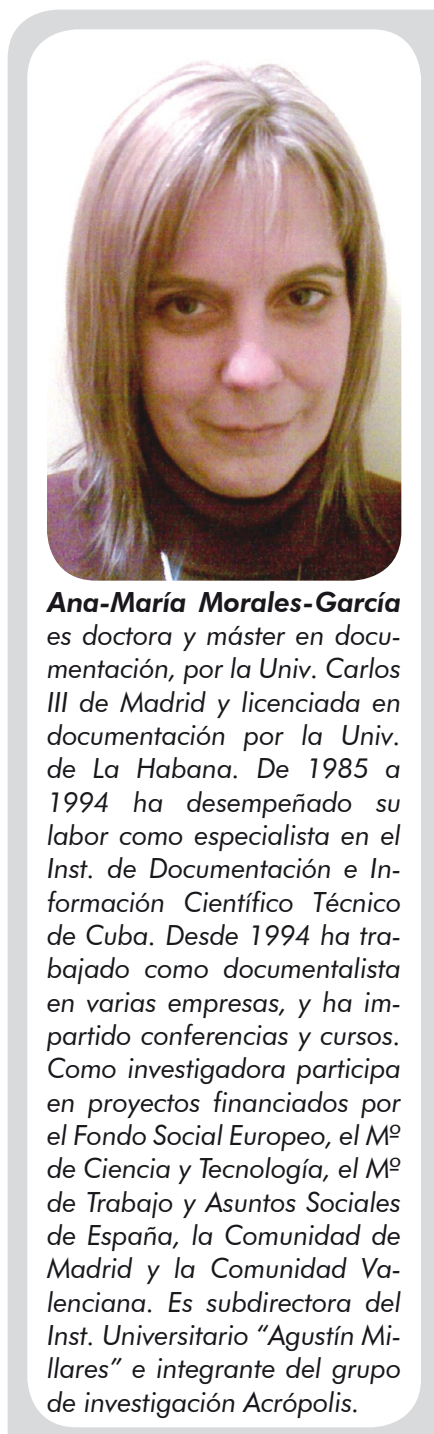

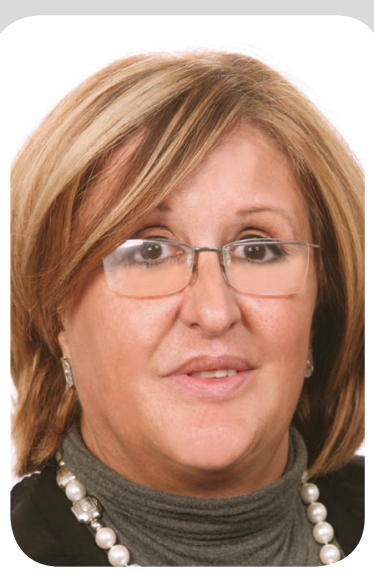

Mercedes Caridad-Sebastián es licenciada en periodismo por la Universidad Complutense de Madrid y doctora en ciencias de la información por la misma universidad; catedrática del departamento de biblioteconomía y documentación de la Universidad Carlos III de Madrid; directora del instituto de investigación "Agustín Millares" de documentación y gestión de la información de la misma universidad, y de la cátedra especial María Moliner de dicho instituto; coordinadora responsable del grupo Acrópolis; y directora del Máster de Información y Documentación de la Universidad Carlos III de Madrid, hoy Máster en Documentación Audiovisual: el Entorno Digital. Gestión del Conocimiento en

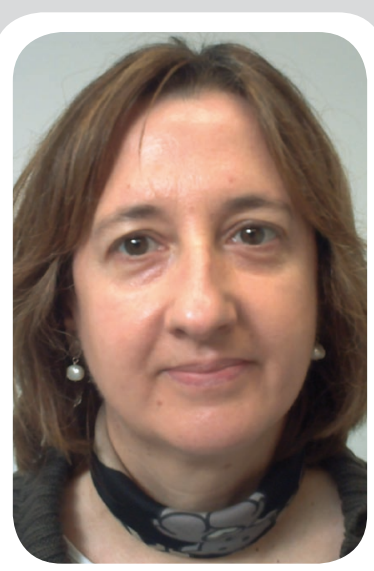

Fátima García-López es linica por la Universidad Autónoma de Madrid y doctora en documentación por la Universidad Carlos III de Madrid. Ha trabajado en diferentes unidades de información y en bibliotecas universitarias. Desde 1998 es profesora en la Universidad Carlos III de Madrid. Entre sus líneas de investigación están las bibliotecas públicas como lugar de integración, los colectivos específicos y los servicios bibliotecarios en comunidades multiculturales. cenciada en filología hispá-

Resumen: Los telecentros españoles constituyen un instrumento de dinamización e inclusión en la sociedad de la información. Sin embargo, no hay análisis que describan las iniciativas que llevan a cabo estas entidades en sus áreas de influencia. Teniendo en cuenta este hecho, se ha realizado una investigación que analiza la situación actual de los telecentros, el fortalecimiento de sus redes y las actividades que realizan. Con este trabajo se propone un "modelo de recursos y servicios" que sirva de soporte para la toma de decisiones por parte de los responsables politicos, o bien, como instrumento de reflexión y análisis para los agentes sociales.

Palabras clave: Telecentros, Puntos de acceso público a internet, Modelo de recursos y servicios, Redes de telecentros.

Title: Telecentres in Spain: networks and content

Abstract: Spanish telecentres are a catalyst and inclusion instrument in the information society. Nevertheless, there is a lack of studies describing the activities performed by these entities in their areas of impact. With this goal in mind, research was conducted to analyse the current situation of the telecentres, the strengthening of their networks and the activities undertaken. It is the goal of this paper to formulate a "resources and services model" as the framework for decision taking by political authorities or as a rethinking and analysis device for social agents.

Keywords: Telecentres, Public internet access points, Resources and services model, Telecentre networks, Content.

Morales-García, Ana-María; Caridad-Sebastián, Mercedes; García-López, Fátima. “Telecentros en España: redes y contenidos". El profesional de la información, 2009, marzo-abril, v. 18, n. 2, pp. 162-170.

DOI: 10.3145/epi.2009.mar.06

\section{Características generales de los telecentros}

En la Unión Europea se están llevando a cabo programas cuyo propósito es eliminar cualquier forma de discriminación social generada por la introducción de las tecnologías de la información y las comunicaciones (TIC). Esta idea se materializa mediante los planes de acción eEurope e i2010 que establecen como prioridad la organización de un programa de puntos de acceso público a internet (PAPI), que permita que todos los ciudadanos dispongan en sus municipios de estos centros, usando preferentemente conexión de banda ancha.

En España, al igual que en el resto de los países, el nombre que se da a este tipo de entidad varía, tomando diferentes denominaciones como: telecentros, nuevos centros del conocimiento (NCC), centros de acceso pú- 
blico a internet (CAPI), KZgunea, o puntos de acceso público a internet (PAPI), que amplían su concepto e incluyen también los centros de enseñanza y las bibliotecas. Las diferencias en la forma de denominarlos están muy ligadas a los servicios que prestan, a las iniciativas que llevan a cabo y a la región de que se trate.

- Telecentros: Es el término más difundido y la manera más común de denominar a estas entidades. Proliferan en España a partir de 1991 con la ayuda de iniciativas comunitarias, programas y planes de acción europeos financiados por los Fondos estructurales. Su propósito no es únicamente la alfabetización digital, sino que suelen cubrir también servicios de formación ocupacional, de asesoramiento tanto a pymes como al público en general así como la puesta en práctica de nuevas formas de trabajo mediante proyectos de teletrabajo. Son fundamentales en zonas rurales o mal comunicadas, pues contribuyen de forma significativa a mejorar su entorno más próximo.

- Nuevos centros del conocimiento (NCC): El proyecto NCC nació en 1999 con el propósito de implantar el Plan de alfabetización tecnológica de Extremadura. Los NCC están ubicados en centros culturales o sociales, donde se ha instalado un equipamiento informático para que todos los sectores de la población adulta tengan acceso a las nuevas oportunidades que las TIC están generando.

- Centros de acceso público a internet (CAPI): Surgen en 2002, y son financiados por los Fondos estructurales y/o iniciativas regionales. Están abiertos a todo el tejido social de los municipios, teniendo en cuenta aquellos grupos que tienen mayor dificultad para el acercamiento a las TIC, mediante acciones formativas (presenciales y online) y talleres de internet. En menor medida proporcionan servicios de asesoramiento personalizado y formación de teletrabajadores.

- KZgunea: Puesta en marcha en 2001 a partir de la colaboración del Gobierno vasco con la Asociación de Municipios Vascos (Eudel), es una red de centros públicos gratuitos para la formación y el uso de las TIC. Los KZgunea ponen internet al alcance de toda la población de Euskadi, centrando su labor en los sectores más alejados de las nuevas tecnologías: amas de casa, jubilados, desempleados e inmigrantes.

- Puntos de acceso público a internet (PAPI): Es el término más genérico y se aplica también a bibliotecas y centros de enseñanza. Tal y como se ha dicho anteriormente, esta terminología ha sido acuñada en los planes de acción eEurope e i2010. Con la ayuda de los PAPI los ciudadanos disponen de fácil acceso a internet en sus municipios, preferentemente a través de banda ancha. Para ello se plantea que los estados miembros utilicen los Fondos estructurales, apoyados con fondos regionales y/o nacionales.

\section{Objeto de estudio y metodología}

En España, el trabajo que venían llevando a cabo los telecentros pasó por una fase que podríamos definir como de "desconocimiento total", no sólo de su existencia, sino del valor social que tiene este tipo de entidad. En este contexto se decidió realizar un primer estudio, que culminó en el año 2001' bajo que finalizó en 2004²; y un seguimiento en 2008 para actualizar y contrastar los datos recopilados.

En esta última investigación nos propusimos no sólo determinar su situación actual, sino formular un "modelo de recursos y servicios" que sirva como soporte para la toma de decisiones por parte de los responsables políticos, o bien, como instrumento de reflexión y análisis para los agentes sociales.

Se plantearon los siguientes propósitos:

1. Valorar la situación de los telecentros españoles.

- Saber su número.

- Determinar la existencia y la labor de las redes nacionales y autonómicas.

- Conocer las iniciativas europeas y regionales que fomentan su creación y sostenibilidad.

2. Proponer un "modelo de recursos y servicios" para los telecentros.

- Identificar los usuarios potenciales.

- Determinar los recursos y servicios.

- Categorizar los recursos y servicios.

La realización del análisis se ha basado, fundamentalmente, en los datos obtenidos de varias fuentes de información:

- Portales de los telecentros españoles.

- Noticias en revistas y periódicos.

- Documentación ofrecida por algunos telecentros y por la entidad Red.es.

- Documentación obtenida en congresos, reuniones, encuentros y seminarios.

- Conclusiones de las entrevistas realizadas a expertos y personal responsable.

\section{Resultados}

\section{Situación de los telecentros españoles}

El apoyo financiero recibido de acciones regionales, nacionales y, sobre todo, comunitarias, ha favorecido el mantenimiento de los telecentros y el fortalecimiento de sus redes. En el transcurso de este estudio se detectó un crecimiento exponencial, pasando de 53 en 2001, a 75 en 2004 y a 6.546 en funcionamiento en 2008, de los cuales 2.995 (un 46\%) están incluidos en una de las 3 redes nacionales (red de telecentros de Red.es, Red 
de Telecentros Rurales y Red Conecta), mientras que 3.551 (un 54\%) pertenecen a una de las 14 redes autonómicas (gráfico 1):

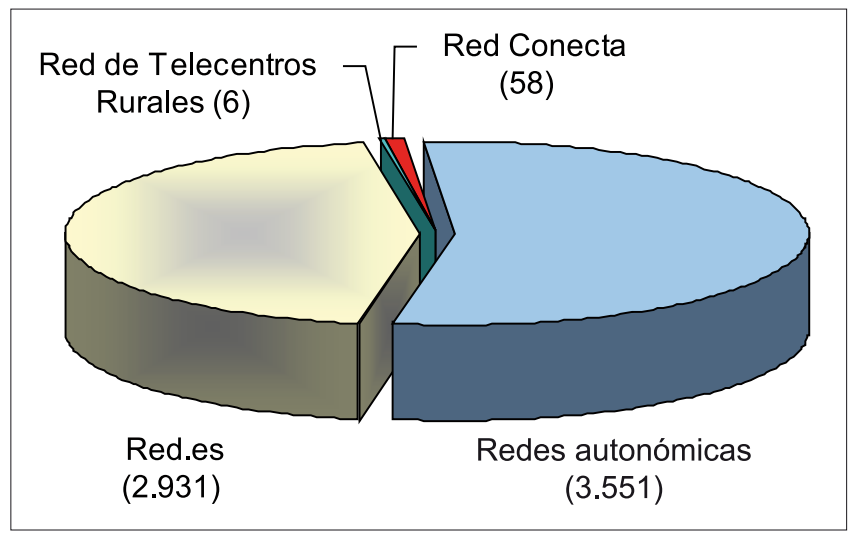

Gráfico 1: Número de telecentros de las redes nacionales y autonómicas

Las 3 redes nacionales son:

- Red.es ${ }^{3}$, con 2.931 telecentros en 14 comunidades autónomas (43 provincias) ${ }^{4}$, es la red de mayor presencia nacional, y surgió a partir de los programas Internet rural y Telecentros.es. Éste intenta establecer las bases para la creación de una futura y única red nacional.

- Red de Telecentros Rurales ${ }^{5}$, en el marco de la iniciativa comunitaria Leader II, ha impulsado la crea-

\section{"Por el número de telecentros en} funcionamiento destacan Castilla y León, Andalucía y Castilla-La Mancha"

ción de 6 telecentros en Bilbao, Navarra, Jaén, Murcia, Vizcaya y Canarias. Trabaja para "compartir, investigar y transferir experiencias y conocimientos, ofreciendo soluciones y servicios tecnológicos a las zonas rurales".

- Red Conecta $^{6}$, con 58 telecentros en 14 comunidades autónomas, es una iniciativa de la Fundación Esplai $^{7}$ que facilita el acceso de determinados colectivos a las TIC y fomenta la igualdad de oportunidades. Esta fundación tiene como objetivos la educación en el tiempo libre de niños y jóvenes, la lucha contra la exclusión social y el desarrollo asociativo.

Por lo que respecta a las 14 redes autonómicas, que agrupan 3.551 telecentros, destacan por el número de entidades creadas Castilla y León (722 telecentros), Andalucía (636) y Castilla-La Mancha (548). La mayoría de centros aún continúan vinculados a iniciativas europeas para el desarrollo social de colectivos desfavorecidos o a políticas de crecimiento rural o urbano, con respaldo autonómico y local.

El gráfico 2 y la tabla 1 muestran la distribución por comunidades autónomas de las 3 redes nacionales

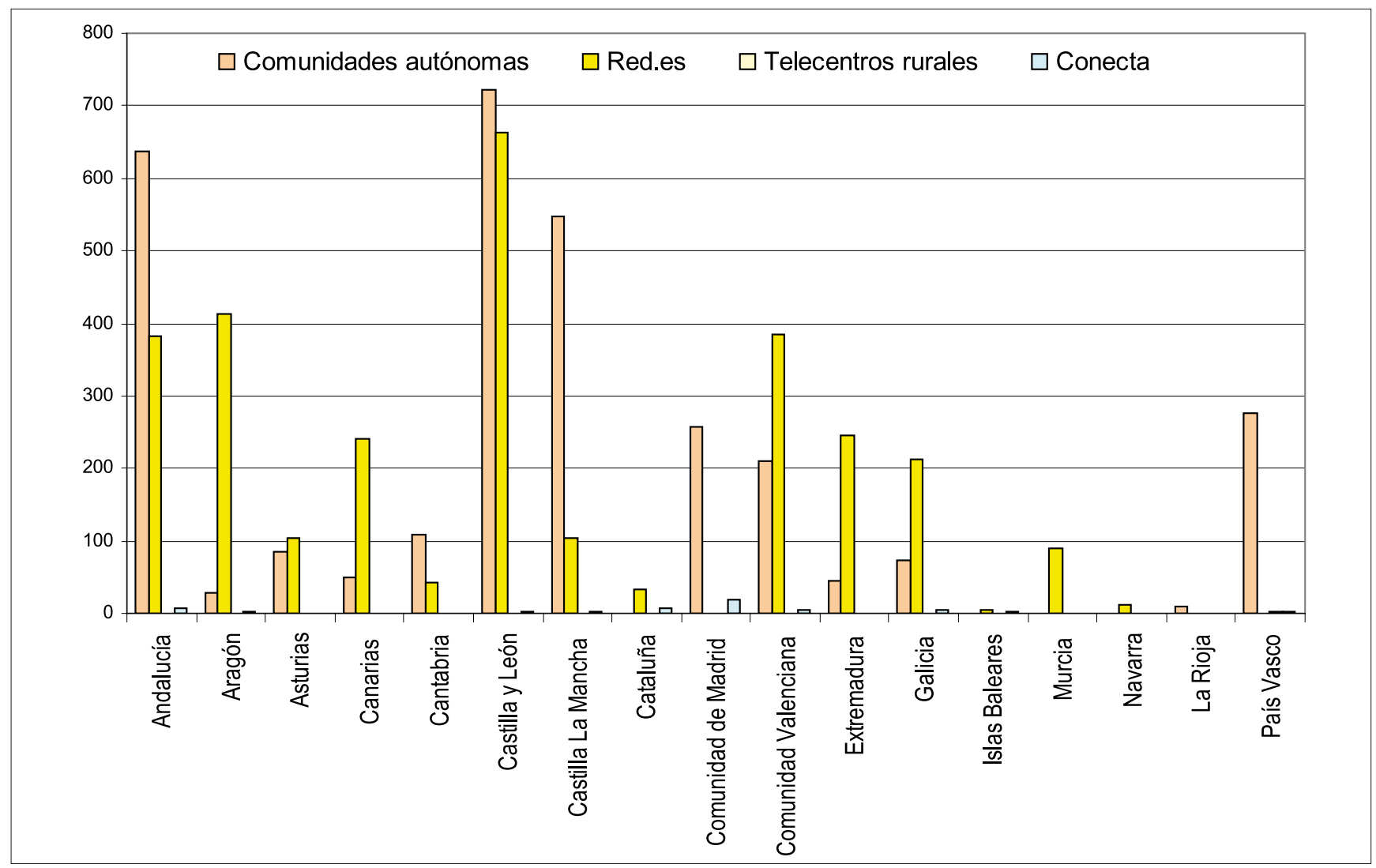

Gráfico 2: Número de telecentros en las comunidades autónomas 


\begin{tabular}{|c|c|c|c|c|c|c|c|}
\hline $\begin{array}{l}\text { Comunidad } \\
\text { autónoma }\end{array}$ & $\begin{array}{l}\text { Redes de telecentros en } \\
\text { comunidades autónomas }\end{array}$ & $\begin{array}{l}\text { Fuentes de } \\
\text { financiación }\end{array}$ & $\begin{array}{l}\text { Redes de } \\
\text { comunidades } \\
\text { autónomas }\end{array}$ & $\begin{array}{l}\text { Red. } \\
\text { es }\end{array}$ & $\begin{array}{c}\text { Red de } \\
\text { Telecentros } \\
\text { Rurales }\end{array}$ & $\begin{array}{c}\text { Red } \\
\text { Conecta }\end{array}$ & Total \\
\hline Andalucía & $\begin{array}{l}\text { Guadalinfo } \\
\text { http://www.guadalinfo.net }\end{array}$ & $\begin{array}{l}\text { Unión Europea (Feder), Junta } \\
\text { de Andalucía, diputaciones } \\
\text { provinciales, ayuntamientos, } \\
\text { Red.es y Red Conecta }\end{array}$ & 636 & 382 & 1 & 8 & 1.027 \\
\hline Aragón & $\begin{array}{l}\text { Centro de servicios labora. } \\
\text { Red de telecentros de Aragón } \\
\text { http://innovacion.ita. } \\
\text { es/labora/ }\end{array}$ & $\begin{array}{l}\text { Programa InnovAragón, } \\
\text { Unión Europea (Feder), } \\
\text { Gobierno de Aragón, } \\
\text { Red.es y Red Conecta }\end{array}$ & 28 & 413 & 0 & 2 & 443 \\
\hline Asturias & $\begin{array}{l}\text { Red de telecentros del } \\
\text { Principado de Asturias } \\
\text { http://www. } \\
\text { asturiastelecentros.com }\end{array}$ & $\begin{array}{l}\text { Unión Europea (Feder), } \\
\text { Principado de Asturias, } \\
\text { Red Conecta y Red.es }\end{array}$ & 85 & 103 & 0 & 1 & 189 \\
\hline Canarias & $\begin{array}{l}\text { Red de telecentros de } \\
\text { Canarias (Retecan) } \\
\text { Web: No disponible }\end{array}$ & $\begin{array}{l}\text { Cabildos insulares y } \\
\text { Red.es }\end{array}$ & 49 & 240 & 1 & 0 & 290 \\
\hline Cantabria & $\begin{array}{l}\text { Red de telecentros de } \\
\text { Cantabria } \\
\text { http://www. } \\
\text { cantabriasitelecentros.com }\end{array}$ & $\begin{array}{l}\text { Gobierno de Cantabria, } \\
\text { ayuntamientos, Red.es }\end{array}$ & 109 & 42 & 0 & 0 & 151 \\
\hline $\begin{array}{l}\text { Castilla y } \\
\text { León }\end{array}$ & $\begin{array}{l}\text { Red de cibercentros de } \\
\text { Castilla y León. } \\
\text { http://cibercentros.jcyl.es/ }\end{array}$ & $\begin{array}{l}\text { Junta de Castilla y León, } \\
\text { ayuntamientos, Red.es } \\
\text { y Red Conecta } \\
\end{array}$ & 722 & 663 & 0 & 3 & 1.388 \\
\hline $\begin{array}{l}\text { Castilla La } \\
\text { Mancha }\end{array}$ & $\begin{array}{l}\text { Red de centros de internet } \\
\text { de Castilla La Mancha } \\
\text { http://www. } \\
\text { centrosinternetclam.com }\end{array}$ & $\begin{array}{l}\text { Unión Europea (Feder), } \\
\text { Junta de Castilla La Mancha, } \\
\text { Red.es y Red Conecta }\end{array}$ & 548 & 104 & 0 & 2 & 654 \\
\hline Cataluña & $\begin{array}{l}\text { Xarxa de telecentres de } \\
\text { Catalunya } \\
\text { http://www.xarxa365.cat }\end{array}$ & $\begin{array}{l}\text { Generalitat de Catalunya, } \\
\text { consejos comarcales, } \\
\text { ayuntamientos, } \\
\text { autofinanciación, Red.es } \\
\text { y Red Conecta }\end{array}$ & 503 & 34 & 0 & 6 & 543 \\
\hline $\begin{array}{l}\text { Comunidad } \\
\text { de Madrid }\end{array}$ & $\begin{array}{l}\text { Centros de acceso público a } \\
\text { internet (CAPI) } \\
\text { http://www.capis.org/ }\end{array}$ & $\begin{array}{l}\text { Programa Innova de } \\
\text { Comunidad de Madrid } \\
\text { y Red Conecta }\end{array}$ & 258 & 0 & 0 & 19 & 277 \\
\hline $\begin{array}{l}\text { Comunidad } \\
\text { Valenciana }\end{array}$ & $\begin{array}{l}\text { Red de puntos de acceso a } \\
\text { internet de la Generalitat } \\
\text { Valenciana } \\
\text { http://papis.gva.es/ }\end{array}$ & $\begin{array}{l}\text { Generalitat Valenciana, Mo } \\
\text { Administraciones Públicas, } \\
\text { Unión Europea (Feder), } \\
\text { Red.es y Red Conecta }\end{array}$ & 210 & 384 & 0 & 5 & 599 \\
\hline Extremadura & $\begin{array}{l}\text { Nuevos centros del } \\
\text { conocimiento (NCC) } \\
\text { http://www.nccextremadura. } \\
\text { org/ }\end{array}$ & $\begin{array}{l}\text { Junta de Extremadura, } \\
\text { Unión Europea (Feder), } \\
\text { ayuntamientos, asociaciones } \\
\text { de vecinos, Red.es y Red } \\
\text { Conecta }\end{array}$ & 45 & 246 & 0 & 1 & 292 \\
\hline Galicia & $\begin{array}{l}\text { Galicia telecentros } \\
\text { http://www. } \\
\text { galiciatelecentros.org/ }\end{array}$ & $\begin{array}{l}\text { Xunta de Galicia, } \\
\text { Red.es y Red Conecta }\end{array}$ & 73 & 213 & 0 & 5 & 291 \\
\hline $\begin{array}{l}\text { Islas } \\
\text { Baleares }\end{array}$ & $\begin{array}{l}\text { Telecentros.es de Red.es } \\
\text { http://www.telecentros. } \\
\text { es/servicios/directorio }\end{array}$ & $\begin{array}{l}\text { Red.es } \\
\text { y Red Conecta }\end{array}$ & 0 & 5 & 0 & 2 & 7 \\
\hline Murcia & $\begin{array}{l}\text { Telecentros.es de Red.es } \\
\text { http://www.telecentros. } \\
\text { es/servicios/directorio } \\
\end{array}$ & Red.es & 0 & 90 & 1 & 0 & 91 \\
\hline Navarra & $\begin{array}{l}\text { Telecentros.es de Red.es } \\
\text { http://www.telecentros. } \\
\text { es/servicios/directorio }\end{array}$ & $\begin{array}{l}\text { Red.es } \\
\text { y Red Conecta }\end{array}$ & 0 & 12 & 1 & 1 & 14 \\
\hline La Rioja & $\begin{array}{l}\text { Fundarco } \\
\text { http://www. } \\
\text { conocimientoytecnologia.org/ }\end{array}$ & $\begin{array}{l}\text { Fundarco, ayuntamientos } \\
\text { y Red Conecta }\end{array}$ & 10 & 0 & 0 & 1 & 11 \\
\hline País Vasco & $\begin{array}{l}\text { Kzgunea } \\
\text { http://www.kzgunea.net/ }\end{array}$ & $\begin{array}{l}\text { Gobierno Vasco, Asociación } \\
\text { de Municipios Vascos (Eudel) } \\
\text { y Red Conecta }\end{array}$ & 275 & 0 & 2 & 2 & 279 \\
\hline Total & & & 3.551 & 2.931 & 6 & 58 & 6.546 \\
\hline
\end{tabular}

Tabla 1: Redes de telecentros nacionales y de comunidades autónomas ${ }^{8}$ 
y las 14 autonómicas. En el total destacan nuevamente por número de entidades en funcionamiento Castilla y León (1.388 telecentros), Andalucía (1.027) y CastillaLa Mancha (654).

En estos momentos representa un reto para todos los telecentros españoles la creación de una única red nacional que coordine todas las iniciativas, con el propósito de que puedan conocerse, comunicarse, saber qué están haciendo, organizar reuniones y solicitar ayudas específicas. El intento de crear esta red nacional se ha visto reflejado en los tres últimos congresos de telecentros celebrados en el País Vasco (2006), Extremadura (2007) e Islas Canarias (2008). Si bien los congresos han constituido un punto de encuentro entre agentes, tanto públicos como privados, dedicados a la optimización de los recursos e infraestructuras de los telecentros de manera cooperativa, hasta el momento el único logro conseguido ha sido la creación, en febrero de 2008, de la asociación Comunidad de Redes de Telecentros.

\section{"El reto ahora es la creación de una única red nacional de telecentros que coordine todas las iniciativas"}

\section{Telecentros: modelización de recursos y servicios}

A pesar de que aún continúan existiendo dificultades relativas al trabajo colaborativo en red, se puede concluir que, en España, los telecentros son mucho más que centros de formación en TIC. Estas entidades se están convirtiendo en dinamizadoras del desarrollo social en zonas desfavorecidas y en muchos casos están llevando a cabo servicios propios de las bibliotecas públicas, y que han sido enunciados en las Pautas sobre los servicios de bibliotecas públicas ${ }^{9}$ y las Pautas para el servicio de acceso a internet en las bibliotecas públicas $^{10}$.

Para comprobarlo se identificaron y evaluaron los servicios y recursos que ofrecen los telecentros a sus usuarios mediante la observación directa de sus portales hasta 2008 .

Nuestra principal herramienta de partida fue el Catálogo de usos, servicios y contenidos ${ }^{11}$, elaborado por Esplai-Fundación Auna a partir de las directrices trazadas en el actual Programa de fomento del uso de internet y las nuevas tecnologías ${ }^{12}$, el cual nos permitió conocer los servicios más comunes en internet.

Partiendo de la metodología empleada en el citado catálogo se han seguido las siguientes etapas de trabajo:

\section{- Usuarios potenciales}

En esta primera etapa, la investigación se centró en identificar a los usuarios que accedían a los recursos y los servicios ofrecidos. Si bien en análisis efectuados hasta el año 2000 existía una fuerte presencia de colectivos específicos (mujeres, autónomos y discapacitados), observamos que el tipo de usuarios se ha ampliado, siendo en estos momentos la práctica totalidad de la población la que puede acceder a todos los servicios, tanto presenciales como virtuales. Sin embargo, sigue estando vigente un particular interés en facilitar servicios a determinados grupos de usuarios a los que

\begin{tabular}{|c|c|}
\hline $\begin{array}{l}\text { Pautas sobre los servicios } \\
\text { de bibliotecas públicas }\end{array}$ & $\begin{array}{c}\text { Pautas para el servicio de acceso a internet } \\
\text { en las bibliotecas públicas }\end{array}$ \\
\hline $\begin{array}{l}\text { La biblioteca pública: } \\
\text { - Es la puerta de acceso a la sociedad de la información y } \\
\text { del conocimiento. } \\
\text { - Garantiza una oferta integral y confluente en información, } \\
\text { formación, ocio y cultura. } \\
\text { - Está abierta a todos los ciudadanos, cualesquiera sean su } \\
\text { condición o actividad. Uno de sus principios fundamenta- } \\
\text { les es el de servir al conjunto de la comunidad y no sólo a } \\
\text { determinados grupos de ciudadanos. } \\
\text { - Desempeña un papel activo en la creación y fomento de } \\
\text { los hábitos de lectura, en la evolución de la creatividad } \\
\text { personal y de la imaginación, y en la utilización del tiempo } \\
\text { libre. Debe ser una entidad estimulante y dinamizadora. } \\
\text { - Tiene una particular responsabilidad en lo relativo a la } \\
\text { recopilación y el fácil acceso a la información local, para } \\
\text { que se mantenga viva la historia de la comunidad a la que } \\
\text { sirve y se fomente la cultura local. } \\
\text { - No debe trabajar aislada, debe buscar fórmulas que le } \\
\text { faciliten su trabajo en red con otras bibliotecas y otros } \\
\text { agentes, con el fin de mejorar la calidad y la amplitud de } \\
\text { los servicios ofrecidos al usuario, y que garanticen asimis- } \\
\text { mo la cooperación, en particular en el entorno local. }\end{array}$ & $\begin{array}{l}\text { Cada biblioteca debería definir los servicios teniendo en } \\
\text { cuenta prestaciones básicas como: navegación www, } \\
\text { uso de correo electrónico e impresión, grabación y des- } \\
\text { cargas de ficheros. } \\
\text { Prestaciones de valor añadido: } \\
\text { - Elaboración de una selección de recursos generales } \\
\text { especializados y de páginas con enlaces selecciona- } \\
\text { dos para niños, mujeres, inmigrantes, etc. } \\
\text { - Orientación y ayuda personal al usuario en el manejo } \\
\text { de las herramientas de navegación. } \\
\text { - Creación de herramientas de ayuda, como guías que } \\
\text { fomenten y faciliten activamente el acceso responsa- } \\
\text { ble de los usuarios a información de calidad. } \\
\text { - Organización habitual de trabajos de formación enca- } \\
\text { minados a que toda la población conozca las utilida- } \\
\text { des de internet y aprenda a utilizar la información } \\
\text { electrónica de un modo eficaz. Se aconseja impartir } \\
\text { cursos de alfabetización informacional, cursos básicos } \\
\text { de búsqueda de información útil en internet, así como } \\
\text { cursos destinados a grupos de usuarios con necesida- } \\
\text { des especiales. }\end{array}$ \\
\hline
\end{tabular}

Tabla 2: Pautas para bibliotecas públicas 
se pretende llegar con más fuerza. Este es el caso de los nuevos emprendedores, las pymes y los colectivos con dificultad de inserción laboral y social (tercera edad, discapacitados, desempleados, inmigrantes, jóvenes y mujeres con déficit cultural).

\section{- Recursos y servicios}

En una segunda etapa, con el fin de identificar y denominar los recursos y servicios prestados, accedimos a cada uno de los 6.546 portales de telecentros, analizando todos sus contenidos. Utilizamos como referencia el Catálogo de usos, servicios y contenidos mencionado anteriormente, y en el caso de que alguno de los servicios no estuviera descrito en el catálogo, tomamos nota de las diferentes denominaciones en cada uno de los telecentros observados, comparándolas con la terminología aportada en las normas ISO/UNE. Esto nos permitió no sólo identificar los servicios, sino homogeneizar las diferentes designaciones.

- Categorización de recursos y servicios

En una tercera etapa, y con todos los servicios identificados, procedimos a clasificarlos y a contabilizarlos por frecuencia de aparición en cada uno de los telecentros. El resultado puso de manifiesto 85 servicios, clasificados en 11 categorías, donde destacaron como los más solicitados: formación y e-formación (100\%), todo lo relacionado con internet $(64 \%)$ y los de información al ciudadano (45\%) (gráfico 3, tabla 3).

De esta forma elaboramos un "Modelo de recursos y servicios" que prestan en este momento todos los telecentros (tabla 3). Con este modelo se ha identificado una serie de servicios y recursos informativos similares a los que prestan, o deberían prestar, las bibliotecas públicas: difusión de alertas, elaboración y difusión de boletines, estadísticas, glosarios, libros, revistas y newsletters, además de las actividades que tienen que ver con la alfabetización informacional, como e-formación y formación. Partiendo de este modelo podemos considerar que los telecentros, al igual que las bibliotecas públicas, son instituciones que prestan servicios de educación, cultura e información democráticamente, es decir, están a disposición de todos los miembros de la comunidad por igual, sea cual sea su edad, sexo, idioma, discapacidad, condición económica, laboral y nivel de instrucción.

Cada una de las 11 categorías que componen este Modelo contiene un promedio de 7 ítems (entre 2 y 19), donde destaca por la diversidad de iniciativas el apartado "Información y servicios al ciudadano" (tabla 3 y gráfico 4).

\begin{tabular}{|c|c|}
\hline \multicolumn{2}{|r|}{ MODELO DE RECURSOS Y SERVICIOS } \\
\hline Categoría general & Descripción \\
\hline $\begin{array}{l}\text { 1. Administración electrónica } \\
\text { (e-administración) }\end{array}$ & $\begin{array}{l}\text { 1. Gestiones y trámites (hacienda, seguridad social, tarjeta sanitaria, licencias de } \\
\text { caza) } \\
\text { 2. Acceso a información sobre leyes y normativas } \\
\text { 3. Atención a inmigrantes, agricultores } \\
\text { 4. Información sobre convocatorias y ayudas } \\
\text { 5. Enlaces a páginas de la e-administración } \\
\text { 6. DNI electrónico }\end{array}$ \\
\hline $\begin{array}{l}\text { 2. Formación } \\
\text { (e-formación) }\end{array}$ & $\begin{array}{ll}\text { 7. } & \text { Formación para adultos } \\
\text { 8. } & \text { Formación profesional } \\
\text { 9. } & \text { Formación de teletrabajadores } \\
\text { 10. } & \text { Formación para discapacitados } \\
\text { 11. Tutorización para acceso a la universidad de mayores de } 25 \text { años } \\
\text { 12. Cursos para niños } \\
\text { 13. Recursos para docentes, dinamizadores o materiales de apoyo } \\
\text { 14. Orientación escolar } \\
\text { 15. Becas } \\
\text { 16. Idiomas para extranjeros } \\
\text { 17. Concursos, talleres, gymkhanas y juegos en la Red }\end{array}$ \\
\hline $\begin{array}{l}\text { 3. Banca on-line } \\
\text { (e-banca) }\end{array}$ & $\begin{array}{l}\text { 18. Trámites comunes } \\
\text { 19. Servicios a pymes } \\
\text { 20. Consultas } \\
\text { 21. Información bursátil }\end{array}$ \\
\hline $\begin{array}{l}\text { 4. Salud on-line } \\
\text { (e-salud) }\end{array}$ & $\begin{array}{l}\text { 22. Concertar visitas } \\
\text { 23. Localización de farmacias de guardia } \\
\text { 24. Localización de centros hospitalarios } \\
\text { 25. Telemedicina } \\
\text { 26. Información y servicios sanitarios } \\
\text { 27. Sexualidad } \\
\text { 28. Drogodependencia } \\
\text { 29. Tercera edad } \\
\text { 30. Información sobre asociaciones de apoyo a enfermos } \\
\text { 31. Trámites }\end{array}$ \\
\hline
\end{tabular}

Tabla 3: Modelo de recursos y servicios 


\section{Conclusiones}

- La mayoría de los telecentros que están funcionando en España han surgido vinculados a iniciativas europeas para el desarrollo social de determinados colectivos desfavorecidos o a políticas de crecimiento rural o urbano, en los que la financiación ha sido el punto de despegue. Las ayudas europeas han estado respaldadas por acciones regionales y nacionales cuya finalidad fundamental es el acercamiento de las TIC a la población española.

- El apoyo financiero ha permitido la creación y mantenimiento de los telecentros españoles y el fortalecimiento de las redes de telecentros. En la actualidad existen 3 redes nacionales y 14 redes autonómicas que suman 6.546 telecentros.

\begin{tabular}{|c|c|}
\hline \multicolumn{2}{|r|}{ MODELO DE RECURSOS Y SERVICIOS } \\
\hline Categoría general & Descripción \\
\hline $\begin{array}{l}\text { 5. Comercio electrónico } \\
\text { (e-comercio) }\end{array}$ & $\begin{array}{l}\text { 32. Compra on-line } \\
\text { 33. Información sobre organizaciones de consumidores y usuarios }\end{array}$ \\
\hline $\begin{array}{l}\text { 6. Información y servicios } \\
\text { al ciudadano }\end{array}$ & $\begin{array}{l}\text { 34. Derechos ciudadanos } \\
\text { 35. Información de partidos políticos, sindicatos, ONGs } \\
\text { 36. Boletines electrónicos } \\
\text { 37. Estadísticas e informes sobre la sociedad de la información } \\
\text { 38. Libros electrónicos } \\
\text { 39. Noticias } \\
\text { 40. Newsletters } \\
\text { 41. Revistas electrónicas } \\
\text { 42. Traducción } \\
\text { 43. Telesecretariado } \\
\text { 44. Gestión de nóminas } \\
\text { 45. Contabilidad } \\
\text { 46. Edición y maquetación } \\
\text { 47. Escaneo } \\
\text { 48. Videoteca } \\
\text { 49. Biblioteca } \\
\text { 50. Sala de reuniones } \\
\text { 51. Asesoría } \\
\text { 52. Glosarios }\end{array}$ \\
\hline 7. Internet y tecnología & $\begin{array}{l}\text { 53. Chat } \\
\text { 54. Correo electrónico } \\
\text { 55. Creación y mantenimiento de blogs } \\
\text { 56. Creación de sitios web } \\
\text { 57. Alertas } \\
\text { 58. Foros } \\
\text { 59. Portales turísticos } \\
\text { 60. Software libre (ejemplo: Linex y Guadalinex, aplicación en tiendas virtuales, } \\
\text { búsqueda de empleo, creación de publicaciones digitales, gestión empresarial) } \\
\text { 61. Descargas } \\
\text { 62. Protección a menores }\end{array}$ \\
\hline $\begin{array}{l}\text { 8. Búsqueda de } \\
\text { información }\end{array}$ & $\begin{array}{l}\text { 63. Búsquedas en internet } \\
\text { 64. Localización de información interna } \\
\text { 65. Búsqueda de telecentros } \\
\text { 66. Transporte por carretera } \\
\text { 67. Medios de comunicación } \\
\text { 68. Consultas lingüísticas } \\
\text { 69. Enciclopedias virtuales }\end{array}$ \\
\hline 9. Empleo & $\begin{array}{l}\text { 70. Información sobre sindicatos } \\
\text { 71. Bases de datos de empresas demandantes } \\
\text { 72. Bolsas de trabajo } \\
\text { 73. Teletrabajo } \\
\text { 74. Derechos laborales } \\
\text { 75. Formación orientada a la inserción laboral }\end{array}$ \\
\hline $\begin{array}{l}\text { 10. Emprendedores } \\
\text { y pymes }\end{array}$ & $\begin{array}{l}\text { 76. Asesoramiento } \\
\text { 77. Listado de colegios profesionales } \\
\text { 78. Búsqueda de empleo } \\
\text { 79. Bolsa de ideas: gestión y proyectos }\end{array}$ \\
\hline $\begin{array}{l}\text { 11. Difusión de actividades } \\
\text { de ocio y cultura }\end{array}$ & $\begin{array}{l}\text { 80. Agenda de ocio } \\
\text { 81. Museos y exposiciones } \\
\text { 82. Cultura popular } \\
\text { 83. Acceso a portales de ocio } \\
\text { 84. Viajes e información (ejemplo: Camino de Santiago) } \\
\text { 85. Enciclopedia virtual para el rescate del patrimonio cultural (ejemplo: Andalucía } \\
\text { Wikanda) }\end{array}$ \\
\hline
\end{tabular}

Tabla 3 (continuación): Modelo de recursos y servicios 


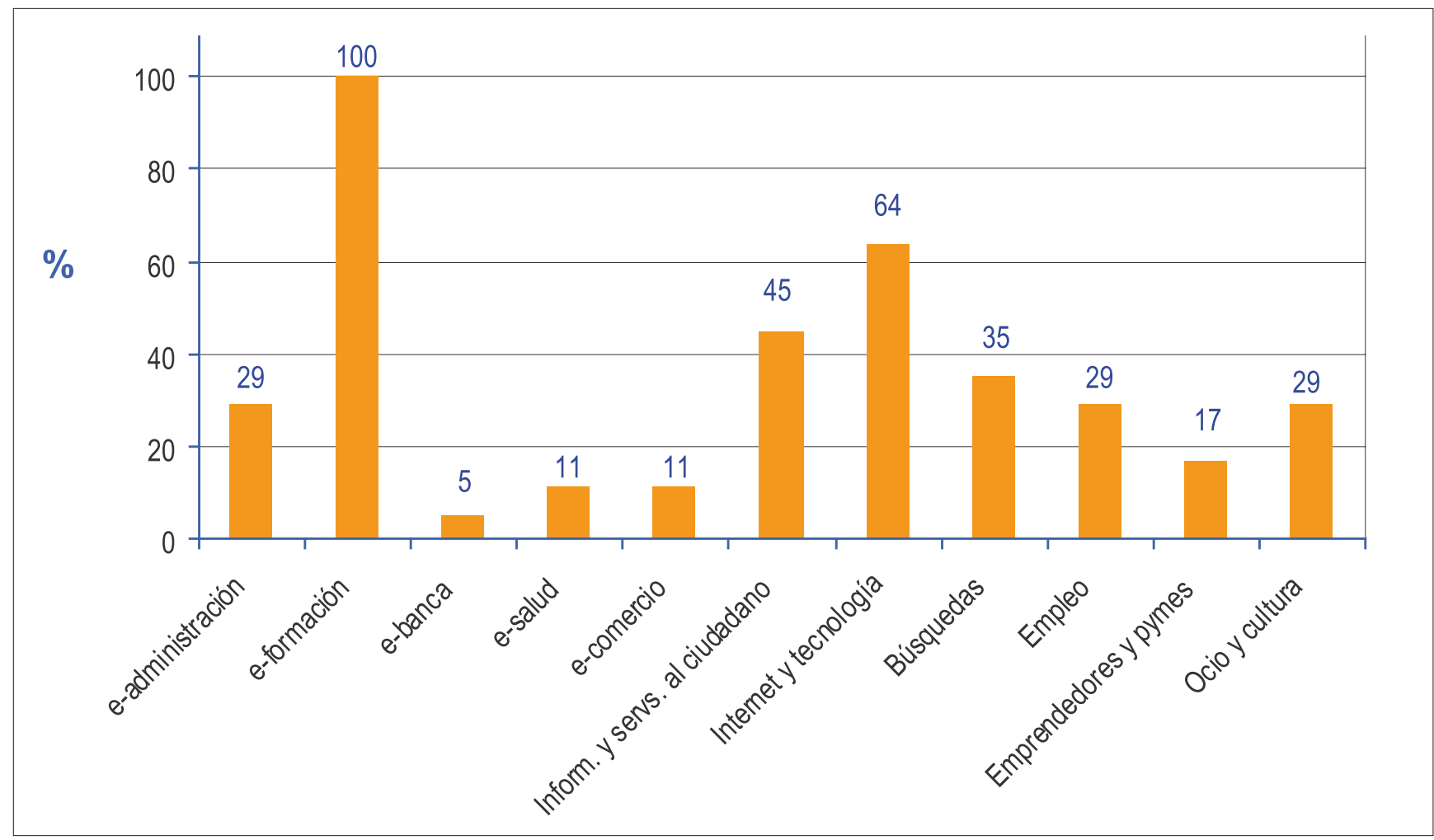

Gráfico 3: Porcentajes de recursos y servicios ofrecidos por los telecentros

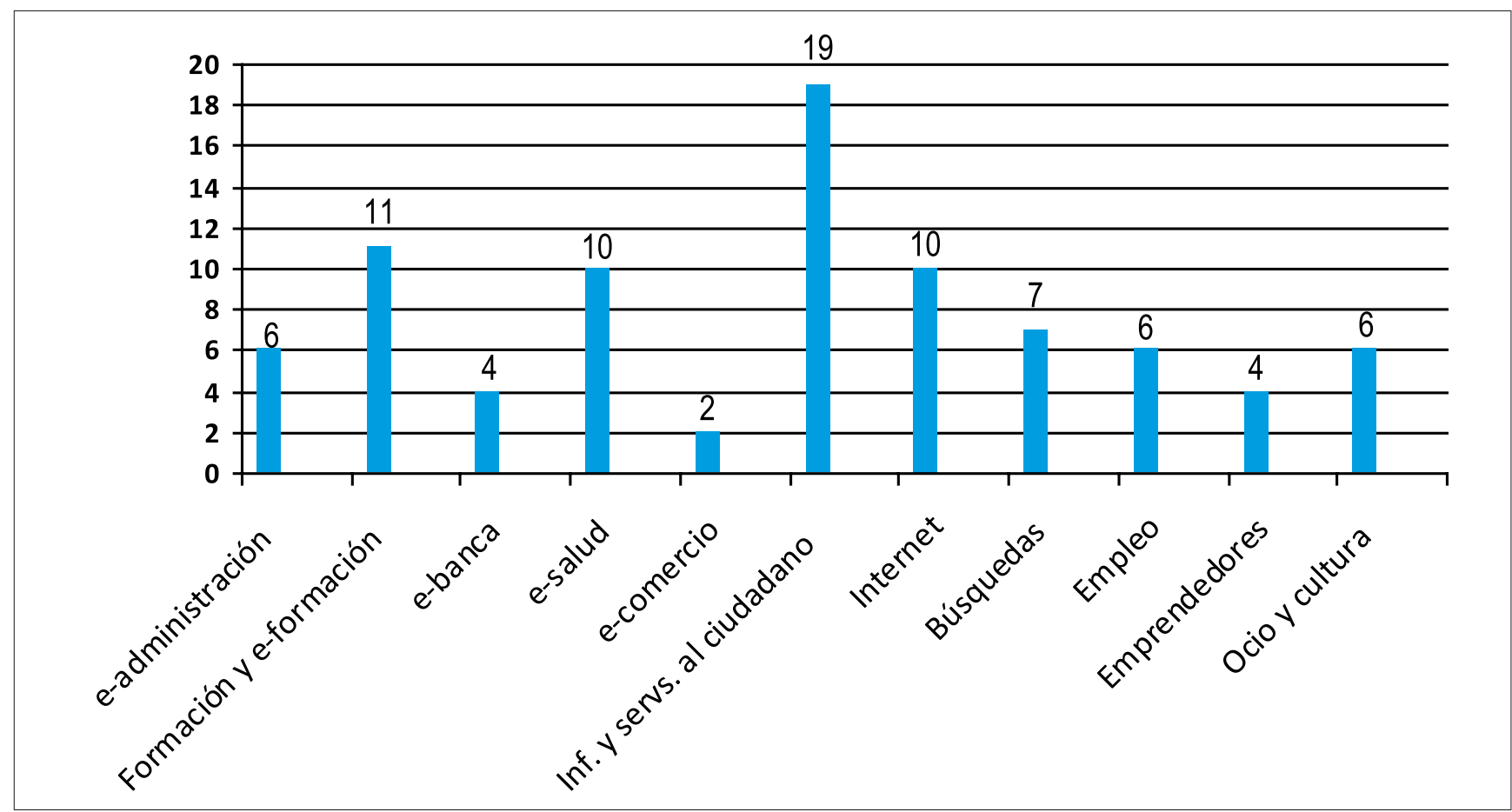

Gráfico 4: Número de servicios en cada categoría

- Los telecentros se están convirtiendo en dinamizadores del crecimiento social en zonas desfavorecidas, y en muchos casos prestan algunos servicios propios las bibliotecas públicas.

- Con los resultados obtenidos se ha elaborado un "Modelo de servicios y recursos". Esta representación de la realidad tiene como propósito fundamental co- nocer la totalidad de los servicios y recursos prestados por los telecentros. Con esta descripción, y con un mayor conocimiento del objeto de análisis, podremos contrastar el porcentaje de recursos y servicios que los telecentros ponen al servicio del usuario, herramienta fundamental para la toma de decisiones por parte de los responsables políticos o bien para la reflexión de los agentes sociales. 


\section{Notas}

1. Morales-García, Ana-María. Sociedad de la información en España: el teletrabajo como acción clave. Tesis doctoral. Madrid: Universidad Carlos III de Madrid, 2002.

http://hdl.handle.net/10016/481

2. Morales-García, Ana-María; Pérez-Lorenzo, Belén. "Telecentros versus NCC y CAPI: herramientas de dinamización de las TIC en la sociedad del conocimiento". En: Caridad-Sebastián, M.; Nogales-Flores, J. T. (coord.): La información en la posmodernidad: la sociedad del conocimiento en España e Iberoamérica. Madrid: Centro de Estudios Ramón Areces, 2004, pp. 325-337.

3. Red.es

http://www.red.es/index.action

4. Los telecentros por dentro y por fuera: análisis y buenas prácticas. Madrid: Red.es, 2008.

http://www.telecentros.es/servicios/utilidades/?contenido=libro-telecentros

5. Red de Telecentros Rurales.

http://telecentrosrurales.com/lared/index.php

6. Red Conecta.

http://www.redconecta.net

7. Fundación Esplai.

http://www.fundacionesplai.org

8. Datos recopilados a partir de entrevistas, de los portales de las redes autonómicas y nacionales, y del libro electrónico elaborado por Karma Peiró, Las redes de telecentros en España. Una historia por contar.

http://www.asturiastelecentros.com/recursos/doc/5823_44442006185537.pdf

9. Pautas sobre los servicios de las bibliotecas públicas. Madrid: Ministerio de Educación Cultura y Deporte, 2002.

http://travesia.mcu.es/documentos/pautas_servicios.pdf

10. Pautas para el servicio de acceso a internet en las bibliotecas públicas Madrid: $\mathbf{M}^{\mathrm{o}}$ de Educación, Cultura y Deporte, 2005.

http://travesia.mcu.es/documentos/pautas_internetbp.pdf

11. Fundación Esplai-Fundación Auna. Elaboración de un catálogo de servicios para fomentar el uso de internet y las nuevas tecnologías. Metodología de trabajo.

http://fomento.telecentros.es/doc/catalogo.pdf (acceso restringido).

12.- El Programa de fomento del uso de internet y las nuevas tecnologías está promovido por la Secretaría de Estado de Telecomunicaciones y para la sociedad de la información, mediante la entidad pública Red.es. Su propósito es contribuir al incremento de usuarios de internet apoyándose en las redes de telecentros existentes en España y mediante la complicidad de empresas, instituciones y organizaciones sociales. La actuación central de este programa consiste en elaborar un catálogo de usos, servicios y contenidos, ligados a las actividades de la vida cotidiana y al uso de las TIC.

http://fomento.telecentros.es/index2.htm (acceso restringido).

\section{Bibliografía}

Fundación Esplai-Fundación Auna. Elaboración de un catálogo de servicios para fomentar el uso de internet y las nuevas tecnologías. Metodología de trabajo.

http://fomento.telecentros.es/doc/catalogo.pdf (acceso restringido).

Morales-García, Ana-María; Pérez-Lorenzo, Belén. "Telecentros versus NCC y CAPI: herramientas de dinamización de las TIC en la sociedad del conocimiento". En Caridad-Sebastián, M.; Nogales-Flores, J. T. (coord.) La información en la posmodernidad: la sociedad del conocimiento en España e Iberoamérica. Madrid: Centro de Estudios Ramón Areces, 2004. pp. 325-337.

Morales-García, Ana-María. Sociedad de la información en España: el teletrabajo como acción clave. Tesis doctoral. Madrid: Univ. Carlos III de Madrid, 2002.

http://hdl.handle.net/10016/481

Morales-García, Ana-María; Ayuso-Sánchez, M.-José. “Telecentros españoles: instrumentos de dinamización del desarrollo social”. En: CaridadSebastián, M. (coord.). Teletrabajo y comercio electrónico en la sociedad de la información. Madrid: Centro de Estudios Ramón Areces, 2001, pp. 103-138.

Morales-García, Ana-María; Caridad-Sebastián, Mercedes; GarcíaLópez, Fátima. "Los telecentros en la sociedad del conocimiento: situación actual y modelo de evaluación". En: I Conf. Intl. "Brecha digital e inclusión social”, celebrada en San José, Costa Rica, 27-29 febr. 2008, organizada por Progr. Soc. de la Inform. y Conocimiento (Prosic) de la Univ. de Costa Rica y el Inst. Univ. A. Millares de la Univ. Carlos III de Madrid.

Pulgar-Vernalte, Francisca; Marcos-Maciá, Sonia. "Utilidades del certificado digital Izenpe en las bibliotecas públicas de Euskadi”. El profesional de la información, 2006, julio-agosto, v. 15, n. 4, pp. 307-309.

http://www.elprofesionaldelainformacion.com/contenidos/2006/julio/9.pdf

\section{Ana-María Morales-García y Fátima García-López Universidad Carlos III de Madrid \\ Calle Madrid, 128. 28903 Getafe \\ amorales@bib.uc3m.es \\ fatimag@bib.uc3m.es}

\section{Mercedes Caridad-Sebastián}

Instituto Universitario Agustín Millares.

Universidad Carlos III de Madrid.

mercedes@bib.uc3m.es

\section{Próximos temas centrales}

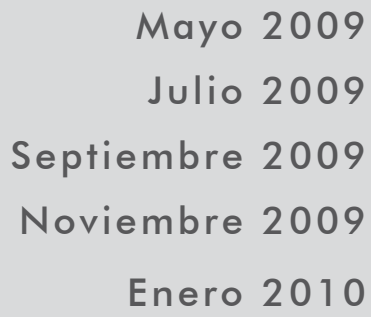

Mayo 2009

Documentación y medios de comunicación Gestión de información personal Información científica y técnica Servicios y sistemas de referencia digital Sector editorial

Los interesados pueden remitir notas, artículos, propuestas, publicidad, comentarios, etc., sobre estos temas a: http://recyt.fecyt.es/index.php/EPI/index 